\title{
Dulaglutide for effective diabetes management
}

\author{
Khan $\mathrm{C}^{*}$ \\ Freelance medical writer, India
}

\begin{abstract}
The clinical and practical consideration of GLP1RAs has risen incredibly to treat T2DM since the development of Dulaglutide- a once-weekly formulation with the combined positive effects on both glucose control and weight management, along with the potential reduction in cardio-renal outcomes.
\end{abstract}

\section{Introduction}

Diabetes Mellitus, commonly known as diabetes, is a prevailing disease that influences the way a body handles glucose, a kind of sugar, in the blood. Most of the population suffers from Type 2 Diabetes condition.

Type 2 diabetes mellitus (T2DM) is increasingly prevalent worldwide and is associated with abdominal obesity in genetically susceptible individuals. T2DM can cause severe vascular complications harming the individual \& reducing life expectancy. To avoid the complications of T2DM a patient-centred approach is recommended with metformin and lifestyle modifications as first-line therapy for most of the individuals. Sulfonylureas, thiazolidinediones, dipeptidyl peptidase-4(DPP-4) inhibitors (gliptins), sodium-glucose cotransporter type 2 (SGLT2) inhibitors (gliflozins), glucagon-like peptide-1 receptor agonists (GLP1RAs) and insulin are currently available for the options as the Second-line agents. Also, fixed combined doses are also available for T2DM as few individuals need combination therapy. The patient characteristic, preferably patient's body weight, and the property of the drug is extremely important and should be taken into consideration while opting for second line agents/combined therapy [1].

For treatment of T2DM GLP1RAs has been developed which improves glucose control with a low risk of causing hypoglycaemia and simultaneously promoting weight loss. GLP1, an incretin hormone, stimulates a decrease in blood glucose levels by insulin secretion and suppresses glucagon secretion in a glucose-dependent manner while delaying gastric emptying and promoting sufficiency. When the native GLP1 is quickly inactivated by the enzyme Dipeptidyl peptidase 4 (DPP-4), GLP1RAs are slightly modified to resist this inactivation. Although, it should be mentioned that GLP1RAs are classified as shortacting and long-acting GLP1RAs which have distinct pharmacokinetic and pharmacodynamic characteristics based on its molecular size. If we consider from a clinical point of view, the most obvious difference found would be that some GLP1RAs are needed to be injected everyday-like exenatide immediate-release, liraglutide, lixisenatide, whereas others can be injected only once weekly-like dulaglutide, semaglutide, albiglutide etc. From diabetic patient's perspective, onceweekly administration instead of daily injection provides considerable convenience and better medication adherence $[1,2]$.

\section{Dulaglutide}

Dulaglutide is basically a genetically engineered protein. It is one of six marketed GLP1RAs available in the market for the treatment of type 2 diabetes mellitus. It is a human GLP1RA, administered once weekly subcutaneously, which is the peculiarity of Dulaglutide [1]. It consists of GLP1 covalently linked to an Fc fragment of human IgG4 (Figure 1) [3]. It can be administered alone or in combination with other medications for diabetes, along with diet and exercise. It helps in lowering the blood glucose levels by stimulating synthesis and secretion of insulin and inhibiting the secretion of glucagon.

\section{Structure}

Dulaglutide molecule contains two identical, disulphide-linked chains, each containing a human GLP1-analog-sequence covalently linked to a modified human immunoglobulin G4 (IgG4) heavy chain fragment $(\mathrm{Fc})$ by a small peptide-linker (Figure 2) [4].

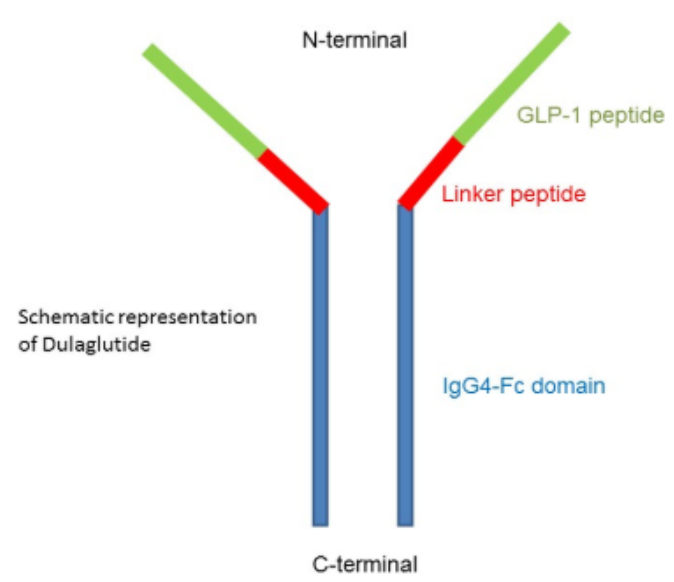

Figure 1. Schematic representation of dulaglutide (Source: Internet)

${ }^{\star}$ Correspondence to: Chandni Khan, Freelance medical writer, India, Tel. 9455705159, E-mail: chandnikhann22@gmail.com

Received: February 18, 2019; Accepted: March 08, 2019; Published: March 12, 2019 


\section{Dulaglutide*: A Once-Weekly GLP-1 Receptor Agonist}

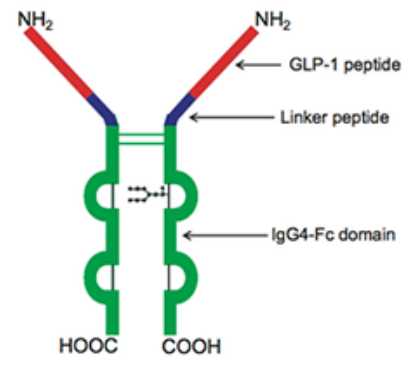

"The US FDA has not yet approved this medication for use.

Figure 2. Structural representation (Source: Internet)

This human GLP1-analog-sequence is approximately $90 \%$ homologous to native-human-GLP1. The amino acid substitutions in Dulaglutide aims at optimizing clinical value like reduced immunogenicity, protection from dipeptidyl peptidase-4 (DPP-4) inactivation and improved solubility of the peptide. The IgG-Fc helps in reducing the rate of clearance and drastically limiting its potential renal excretion by increasing the size of dulaglutide $[1,2]$.

\section{Clinical efficacy}

The clinical efficacy of dulaglutide includes its glucose-lowering activity, postprandial glucose plasma concentrations and $\mathrm{HbAlc}$ levels combined with the body weight reducing effect, which has been carefully investigated in patients with T2DM previously [1].

\section{Drug formulation and dosing}

For commercial use, Dulaglutide has been defined to be $0.75 \mathrm{mg} / 0.5$ $\mathrm{mL}$ or $1.5 \mathrm{mg} / 0.5 \mathrm{~mL}$ solution in a prefilled automatic injection device. The product doesn't needs reconstitution, in contrast to exenatide extended-release. It can be injected in the upper arm, thigh or abdomen region, once in a week, subcutaneously. The dose can be increased, depending on glycemic control. Although for potentially vulnerable populations, i.e. patients $>75 \mathrm{yrs}, 0.75 \mathrm{mg}$ once-weekly is considered as an initial dose. Also, if any dose is missed, it can be administered within 3 days. Dulaglutide is not recommended in severe renal impairment as there isn't sufficient data for this group yet [1].

\begin{tabular}{|c|c|c|}
\hline Drug & Dosage & Storage \\
\hline Dulaglutide & $\begin{array}{c}0.75 \mathrm{mg} / 0.5 \mathrm{~mL} \text { or } 1.5 \mathrm{mg} / 0.5 \mathrm{~mL} \text { for } \\
\text { subcutaneous injections }\end{array}$ & Upto 14 days at RT \\
\hline
\end{tabular}

\section{Side effects and safety}

The most common adverse events associated are transient gastrointestinal disturbances such as nausea and diarrhea, with low inherent risk of hypoglycaemia $[1,2,5]$. There is no increase in cardiovascular problems or mortality when compared with placebo or other therapies available for diabetes, including glargine insulin, sitagliptin and metformin. However, GLP1RAs should not be recommended for patients with pancreatitis history, as per the post marketing reports [6-8].

\section{Conclusion}

Dulaglutide has been approved by the US FDA and the EMA for the management of hyperglycemia in T2DM and $0.75 \mathrm{mg}$ to $1.5 \mathrm{mg}$ once-weekly dose have demonstrated Haemoglobin Alc reduction of $\sim 0.7 \%-1.6 \%$ as monotherapy and in combination (dual or triple therapy) in controlled clinical trials $[9,10]$. The glucose-lowering activity with dulaglutide involves both postprandial plasma glucose concentrations, with low hypoglycaemia risk and reductions of fasting. Similar to other GLP1RAs, It has shown quite rapid and sustained weight loss of $\sim 1.5-3 \mathrm{~kg}$, contrasting with a weight increase with other GLP1RAs. In conclusion, dulaglutide is novel option which is effective, easy for use and well tolerated and it should be located alongside other long-acting GLP1RAs within the recommended guidelines [11-14].

\section{References}

1. Scheen AJ (2016) Dulaglutide (LY-2189265) for the treatment of type 2 diabetes Expert Rev Clin Pharmacol 9: 385-399. [Crossref]

2. Burness CB, Scott LJ (2015) Dulaglutide: A review in type 2 diabetes. BioDrugs 29 407-418. [Crossref]

3. Pace E, Tingen J (2017) Dulaglutide (Trulicity) for type 2 diabetes mellitus. Am Fam Physician 96: 540-542. [Crossref]

4. Jimenez-Solem E, Rasmussen MH, Christensen M, Knop FK (2010) Dulaglutide, a long-acting GLP-1 analog fused with an $\mathrm{Fc}$ antibody fragment for the potentia treatment of type 2 diabetes. Curr Opin Mol Ther 12: 790-797. [Crossref]

5. Scheen AJ (2017) Dulaglutide for the treatment of type 2 diabetes. Expert Opin Biol Ther 17: 485-496. [Crossref]

6. Goudochnikov VI (2018) Role of glucocorticoids and Stress in pathogeny of diabetes mellitus and related disorders. J Diabetes Metab Disord 5: 022

7. Trager G (2018) Should the diabetes therapy recommendations be totally revised? $J$ Diabetes Metab Disord 5: 023.

8. Nayak BS, Sakhamuri SM, Moore I, Jordan D, Cuffie E, et al. (2016) Relationship of lifestyle, quality of sleep with type 2 diabetes and cardiovascular Risk - A Trinidadian study. J Diabetes Metab Disord 3: 013.

9. Merja K, Tarja L, Seppo S, Tuula V, Markku H, et al. (2015) Association between increased insulin resistance index HOMA-IR and COPD in a nationally representative population sample. J Pulm Med Respir Res 1: 001.

10. Tiki T (2017) Prevalence and associated factors of anxiety among type 2 diabetes mellitus patients on follow up at ambo general hospital, oromia regional state, Ethiopia, Institutional based cross sectional study. J Psychiatry Depress Anxiety 3: 007.

11. Fiorini F, Sessa F, Congedo E, De Cosmo G (2015) Diabetes: A continuous challenge for anesthesiologist. J Diabetes Metab Disord 2: 006.

12. Subasinghe SS, Bongetti E, O’Brien CL, Silberberg C, Ward G, et al. (2015) A review of the prevalence and associations of depression and anxiety in type 1 diabetes mellitus. J Diabetes Metab Disord 2: 007.

13. Boonsatean W, Dychawy Rosner I, Carlsson A, Östman M (2016) The influences of income and education on the illness perception and self-Management of Thai adults with type 2 diabetes. J Diabetes Metab Disord 3: 017.

14. Khan C (2018) Phytic acid: boon or curse. J Phytochemistry Biochem 2: 108

Copyright: (C2019 Khan C. This is an open-access article distributed under the terms of the Creative Commons Attribution License, which permits unrestricted use, distribution, and reproduction in any medium, provided the original author and source are credited. 